Techniques \& Culture

\title{
La vannerie saharienne se métisse
}

Saharan basketwork gets crossbred

\section{Tatiana Benfoughal}

\section{OpenEdition}

\section{Journals}

Édition électronique

URL : https://journals.openedition.org/tc/6402

DOI : $10.4000 /$ tc. 6402

ISBN : 1952-420X

ISSN : 1952-420X

\section{Éditeur}

Éditions de l'EHESS

\section{Édition imprimée}

Date de publication : 15 juin 2012

Pagination : 194-211

ISBN : 2-7351-1512-7

ISSN : 0248-6016

\section{Référence électronique}

Tatiana Benfoughal, «La vannerie saharienne se métisse », Techniques \& Culture [En ligne], 58 | 2012, mis en ligne le 30 octobre 2012, consulté le 29 septembre 2022. URL : http://journals.openedition.org/ tc/6402 ; DOl : https://doi.org/10.4000/tc.6402 


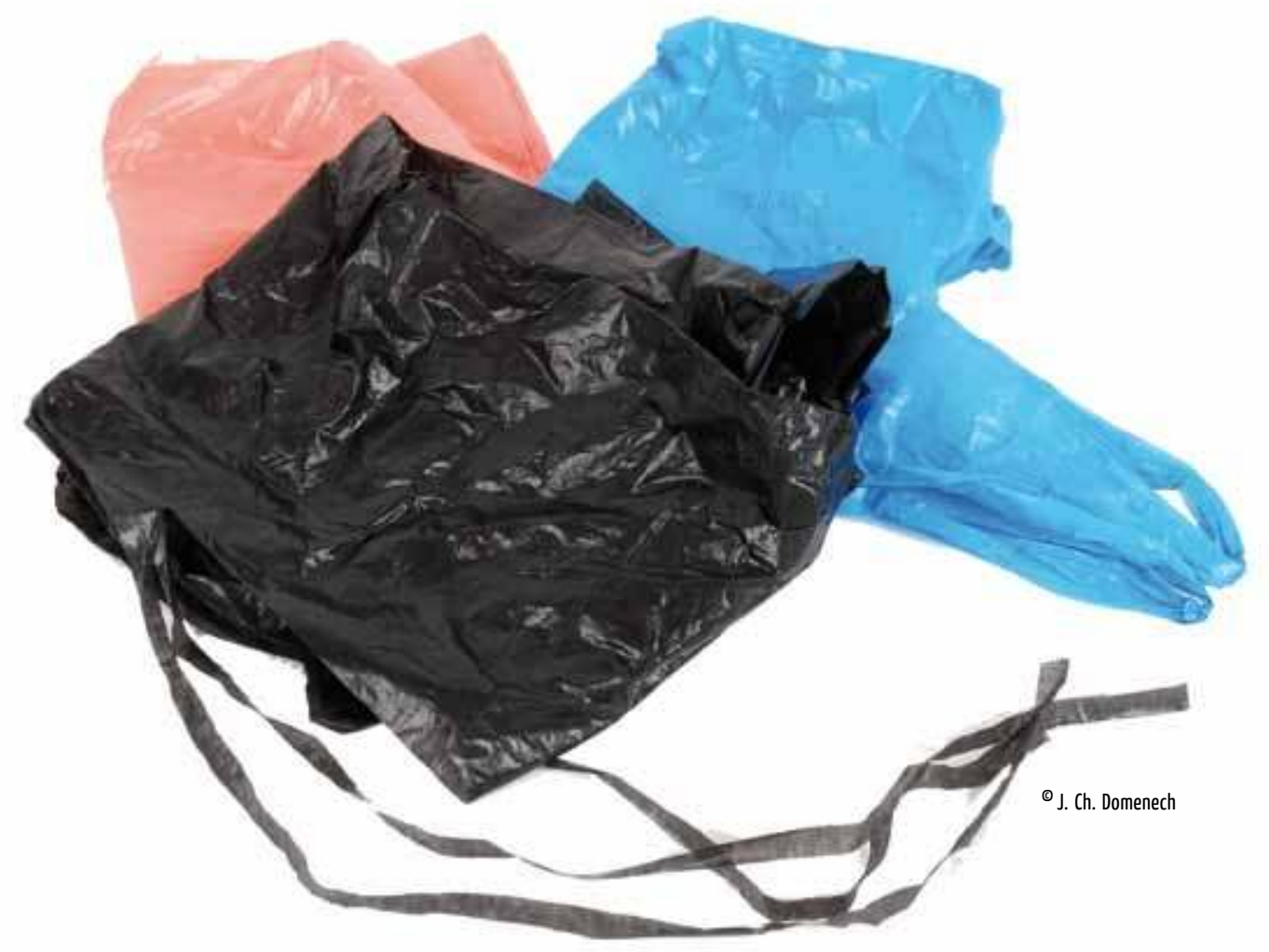




\section{Tatiana Benfoughal}

\section{LA VANNERIE SAHARIENNE SE MÉTISSE}

Depuis les années 1970-80, une vannerie d'un genre nouveau a fait son apparition dans les oasis du Sahara maghrébin ${ }^{1}$. Fabriquée non plus à partir de matières végétales, mais avec du plastique, elle a connu un engouement considérable, comme déjà dans les années 1960 dans tout le Maghreb septentrional ainsi qu'au Proche et Moyen-Orient et plus tard en Afrique sub-saharienne. Ce sont surtout les femmes qui animent cette production, tressant les vanneries chez elles, pour leur propre consommation et partiellement pour la vente à usage local. Leur prix sur les marchés, relativement élevé par rapport aux vanneries en fibres du palmier dattier ${ }^{2}$, reflète la haute valeur que les femmes leur accordent.

Dans ma précédente publication sur le sujet, j’ai utilisé le concept de mode pour analyser le succès des vanneries en matière plastique dans le Sahara algérien (Benfoughal 1996). Effectivement, les mécanismes de diffusion de ces nouvelles vanneries m’ont paru correspondre aux caractéristiques propres au phénomène de mode : la présence de «leaders » (jeunes filles et jeunes femmes), le sens de l'expansion sociale (des couches les plus favorisées à celles qui le sont moins), la rapidité et le sens de la diffusion géographique (à partir des villes vers les zones périurbaines et les petits bourgs, puis vers les régions rurales) et les mécanismes de diffusion (imitation, compétition). Le caractère de mode inhérent à l'introduction de la matière plastique dans le tressage des vanneries impliquait aussi un essoufflement assez rapide du phénomène, ce dont j'étais persuadée sans pouvoir l'affirmer faute de recul suffisant. J'ai pensé qu'après quelques années, la matière plastique, à la portée de tous et banalisée, ne présenterait aucun intérêt aux yeux des femmes, que d'autres matières et d'autres symboles viendraient la remplacer et que la «nouvelle Mode refusera dogmatiquement la Mode qui l'a précédé » (Barthes 1967 : 274). 
Contrairement à mes pronostics, mes dernières missions dans les oasis sahariennes (années 2002-2009) ont montré que non seulement la matière plastique est encore présente dans la production vannière, mais que son importance aujourd'hui est telle qu'elle a atteint, par contagion, presque toutes les oasis en Algérie, au Maroc et en Tunisie.

S'il n'y a pas lieu de

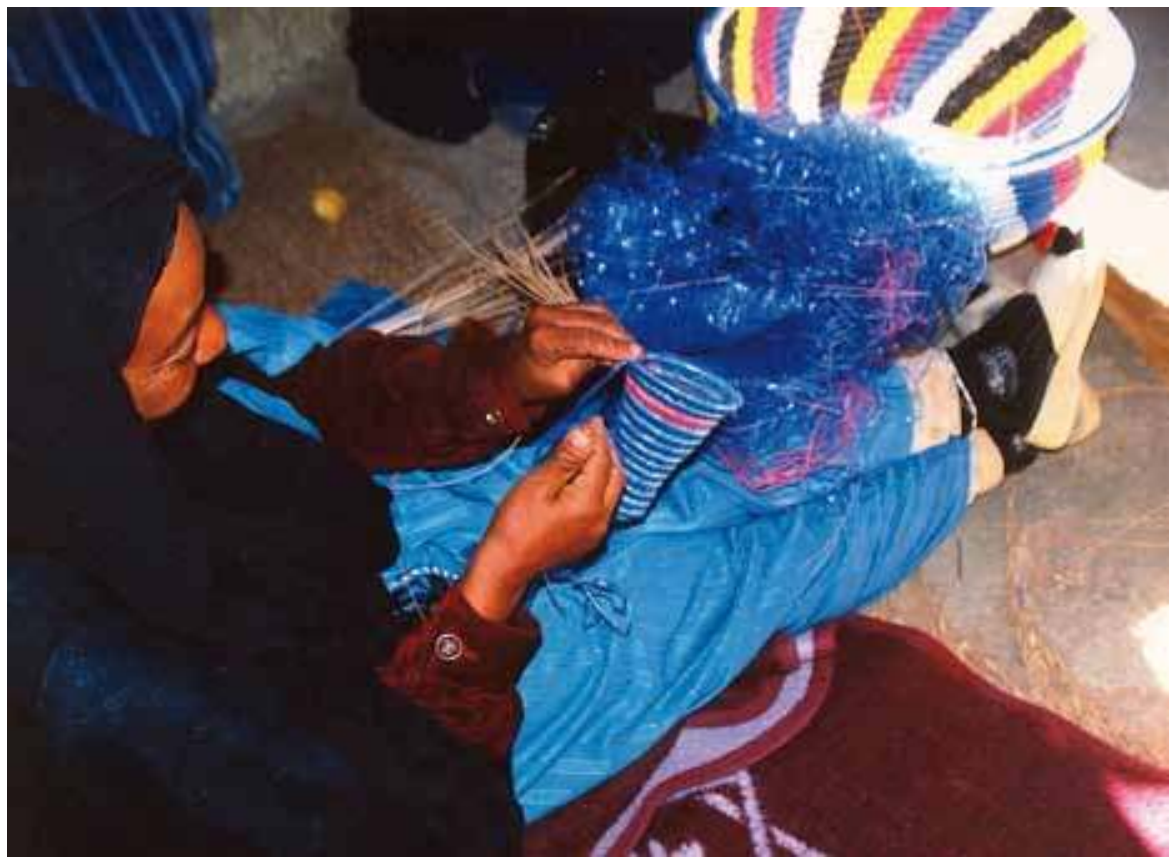

${ }^{\circ} \mathrm{T}$. Benfoughal
Tressage d'une vannerie avec des lanières en matière plastique tirées d'un sac à semoule. Maroc, région de Tata, ksar Aït-Yacine, 2002. contextes d'utilisations des vanneries en matière plastique, qui pouvaient permettre de mieux comprendre leur longévité et surtout de les envisager non pas comme un phénomène isolé, né d'une mode passagère, mais comme la résultante d'un processus durable qui s'inscrit dans une logique socio-économique et culturelle contemporaine. Pour ce faire, j'ai dû recourir à plusieurs concepts parmi ceux avancés par nombre d'auteurs ayant travaillé sur la dynamique des phénomènes culturels et plus particulièrement des objets $^{3}$. Le premier qui m’a paru opérationnel est celui du « métissage culturel », défini comme « la mise en contact de deux identités culturelles entre lesquelles se produisent des échanges et des mélanges » (Turgeon 2003). La plupart des auteurs qui ont travaillé sur ce concept (Laplantine \& Nouss 1997 ; Gruzinski 1999 ; Amselle 2010 ; Bonniol 2001) s'accordent à dire que si le métissage culturel traverse la profondeur historique des sociétés, dont il manifeste le dynamisme, il acquiert cependant des caractéristiques particulières à l'époque moderne et post-moderne. À la suite de la colonisation, de l'industrialisation et du développement du capitalisme marchand, les cultures réceptrices se trouvent submergées par les produits importés d'Europe, puis plus récemment d'Asie, et sont donc en possession de nombreux éléments exogènes à « domestiquer ». De leur côté, les pays industrialisés sont soumis aux courants d'immigration et s'ouvrent de plus en plus à l'influence des cultures non occidentales. On peut dire que désormais 
« aucun secteur du monde intellectuel, artistique et commercial n'échappe à l'utilisation des notions de métissage, d'hybridité et de créolisation » (Amselle 2010 : 12).

Les terrains de métissage sont nombreux et diversifiés, ce qui suppose que les processus d'intégration des éléments endogènes et exogènes ne sont pas les mêmes pour les immigrés dans leur pays d'accueil et pour les autochtones qui reçoivent des éléments culturels exogènes chez eux; pour les élites intellectuelles ou artistiques et le reste de la population; pour le métissage des langues, des savoirs, des pratiques festives ou des techniques artisanales. Ayant observé et étudié pendant presque vingt ans, dans les années 1980-1990, la culture algérienne « de l'intérieur » et ayant eu l'opportunité de poursuivre cette recherche, entre 2002 et 2009, dans les deux pays maghrébins voisins, le Maroc et la Tunisie, j'ai dû effectuer plusieurs choix. J'ai pris d'abord en exemple un cas de métissage où la culture « traditionnelle », en l'occurrence saharienne, accueille un élément exogène chez elle. Ceci m'a conduit à prendre appui sur le point de vue du « récepteur » pour analyser sa manière de « métisser ». Sur le plan thématique, la vannerie m’a paru représentative de nombreux métissages techniques, esthétiques, économiques et identitaires caractérisant actuellement plusieurs artisanats maghrébins et sahariens.

Pour analyser les matières plastiques dans leurs étapes d'adoption et d'adaptation, il m'a semblé aussi intéressant de croiser le concept de métissage avec celui d'emprunt. En effet, celui-ci induit la nécessité d'étudier les mécanismes de métissage du point de vue du récepteur, il impose d'analyser les conditions d'accueil et il met l'accent sur le caractère actif du processus de la réception. Elaboré par A. Leroi-Gourhan (1992) et revisité récemment (Turgeon 2003 ; Rouillard, 2007), ce concept implique la prise en compte non seulement de la nature de l'élément exogène, mais aussi et surtout du «sort qui lui est fait par le milieu intérieur» (Leroi-Gourhan 1992 : 357). Alors plus que « donner », « recevoir» ou « rendre », c'est l'idée de «prendre » qui retiendra notre attention (Turgeon $2003: 23$ ).

\section{Éléments « en métissage »}

Puisque par définition un objet «métissé » est composé d'éléments issus de cultures différentes en situation de contact, son analyse impose de prendre en considération tous ces éléments, qu'ils fussent endogènes et exogènes. Dans le cas qui nous préoccupe, il s'agit de la culture saharienne et de la culture occidentale qui donnent au « métissage », pour la première, des matériaux naturels (végétaux, cuir, laine), des techniques de tressage (spiralé cousu, cordé, tissé ${ }^{4}$ ), des formes fonctionnelles (paniers, couffins, plats, corbeilles, etc.) et certains décors, et pour la deuxième, la matière plastique. Les éléments endogènes sont propres à la vannerie traditionnelle dans un espace géographique qui couvre pratiquement tout le Sahara et se rapportent aux pratiques de tressage des vanneries en fibres issues essentiellement du palmier dattier. Les origines de l'élément exogène sont multiples. Au début de son utilisation, la matière plastique provenait de l'Europe, sous la forme de sacs ou de sachets d'emballage de produits importés. À partir des années 1970, avec le développement des industries nationales locales, le plastique, sous la forme de granulés ou de produits divers, a commencé à se répandre à partir des usines implantées autour des capitales des trois pays maghrébins et des grandes villes, ainsi que dans les 
Tressage d'un panier en matières végétales selon la technique du spiralé cousu

Le principe de cette technique traditionnelle consiste à monter la vannerie à partir du centre, en utilisant ses deux éléments constitutifs : le montant, qui forme l'âme de la vannerie en s'enroulant en spirale, et le brin, élément mobile et souple, destiné à entourer le montant par des enroulements successifs et à fixer les spires entre elles. Le montant se compose de plusieurs tiges de la hampe du régime de dattes défibrée que l'on assemble en faisceau, alors que le brin est constitué de lamelles découpées à partir des folioles de palmes. La femme utilise l'alêne métallique pour percer la spire déjà formée et y introduire le brin de la foliole par son extrémité pointue. Ce travail de fixation des deux spires entre elles par des points de couture (d'où l'appellation de spiralé cousu) se fait à chaque tour du brin autour du montant. Algérie, Tassili n-Ajjer, Djanet, 2008.

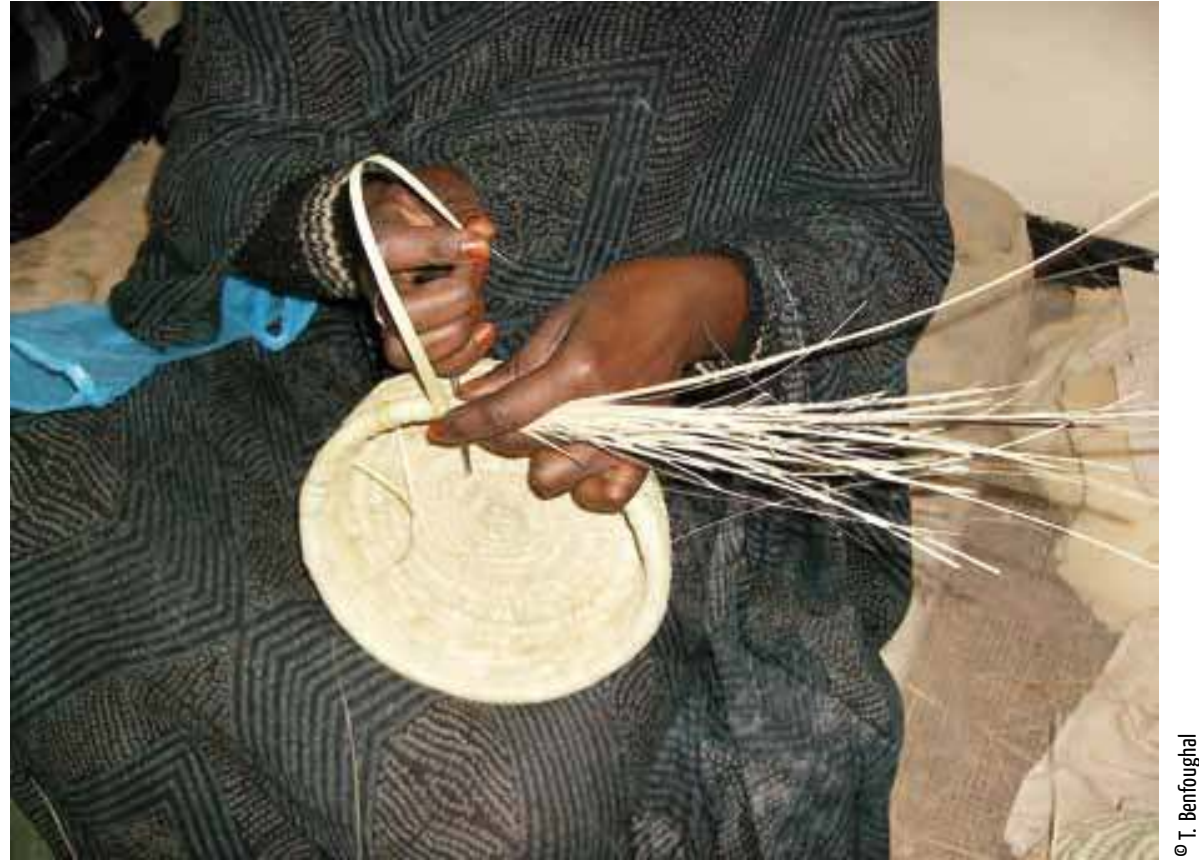

pays de l'Afrique sub-saharienne ${ }^{5}$. Depuis quelques années, le «monde du plastique » s'est rapproché des oasis. Des PME spécialisées notamment dans la fabrication des sacs ou des nattes s'installent aujourd'hui dans les régions sahariennes et présahariennes.

Le processus de mise en commun des éléments endogènes et exogènes suppose une démarche mentale active de la part des vannières. Elle consiste tout d'abord à établir une association entre les propriétés physiques de la matière plastique et son éventuelle utilisation dans le tressage des vanneries. Les expériences accumulées de consommation quotidienne du plastique, tel qu'il était proposé par les industries des années 1970-1980 ont permis aux Sahariens d'en relever les qualités de plasticité, de solidité et de souplesse. Mais, pour passer à son utilisation dans le tressage, il fallait mettre en relation la nouvelle matière et la vannerie. Il fallait imaginer que les folioles du palmier dattier utilisées comme brins pouvaient être remplacées par les rubans de matières plastiques et que l'on pouvait tirer un profit pratique et esthétique des caractéristiques de ces nouvelles matières que la matière végétale traditionnelle ne pouvait pas offrir.

Matières plastiques détournées pour le tressage des vanneries ${ }^{6}$ :

- petits sacs de polyéthylène issus du commerce de détail

- emballages des produits divers (de thé, de lait, de chocolat, etc.) en polyéthylène et en polyéthylène doublé de papier ou de papier aluminium

- grands sacs tissés industriellement à partir d'étroits rubans de polypropylène et destinés à la vente en gros de farine ou de semoule aux détaillants épiciers

- nattes ou lés tissés industriellement à partir de fils tubulaires de polypropylène

- cordes et rubans de polypropylène

- fils de polyamide (nylon) 
Si la possibilité d'utiliser des matières plastiques dans le tressage des vanneries peut être considérée comme une découverte collective, tant il est difficile de dire aujourd'hui par qui, quand et où elle a commencé, les choix de telle ou telle matière plastique, en fonction du type de vannerie à tresser, nécessite une démarche active individuelle chaque fois renouvelée. Le choix individuel des matières s'effectue à l'intérieur de la gamme des produits mis sur le marché par les industries et les commerces de chacun des trois pays maghrébins.

S'agissant d'une matière prélevée sur un objet initial pour un nouvel emploi, nous serions tentés de classer ce moyen d'approvisionnement dans la catégorie de récupération. Mais est-ce réellement le cas? Si on se réfère au sens du terme de récupération, défini comme « action de récupérer ce qui a été perdu, abandonné, détruit »(Petit Robert), ce qui suppose la gratuité, il ne conviendrait qu'à une partie des matières plastiques, celles que les femmes prélèvent des sachets d'emballage des produits de consommation courante, achetés pour les besoins de leurs familles. Mais même dans ce cas, la gratuité est toute relative. Les femmes considèrent que puisque les produits sont achetés, leurs emballages le sont aussi. D'autres matières plastiques sont achetées en tant que telles. Ce mode d'approvisionnement diffère de celui pratiqué par quelques rares PME ou ONG créées en Afrique suite aux initiatives privées, européennes ou locales, et spécialisées dans la fabrication des sacs, cabas et autres accessoires féminins. Dans ces entreprises, animées par l'idée de protection de l'environnement et d'amélioration de la condition des femmes, le travail des ouvrières va du ramassage des sacs plastiques dans les rues jusqu'à leur recyclage en produits crochetés ou tricotés et commercialisés en partie dans les boutiques des grandes villes européennes ${ }^{7}$. Les femmes des oasis, elles, cherchent à utiliser la matière plastique neuve et en tout cas en très bon état, car pour faire de « belles » vanneries, la matière plastique ne doit être ni froissée, ni déchirée, ni décolorée $^{8}$. De fait, celle-ci ne provient jamais des poubelles ni des décharges, elle n’est

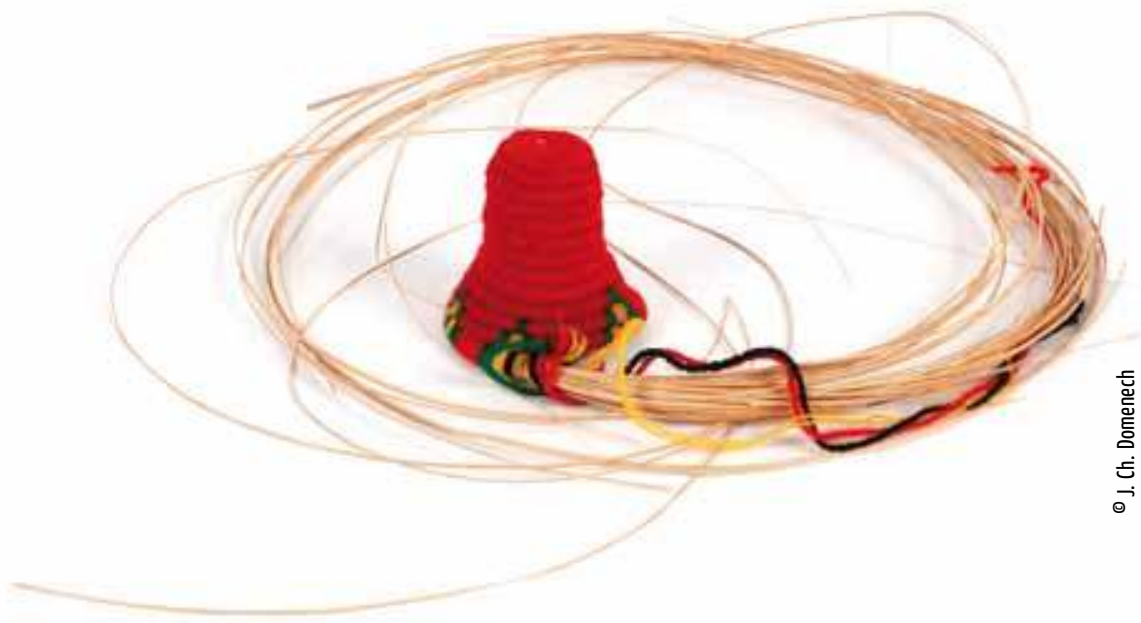

Tressage d'un couvre-plat Le tressage se fait avec des fils de laine selon la technique du spiralé cousu. 


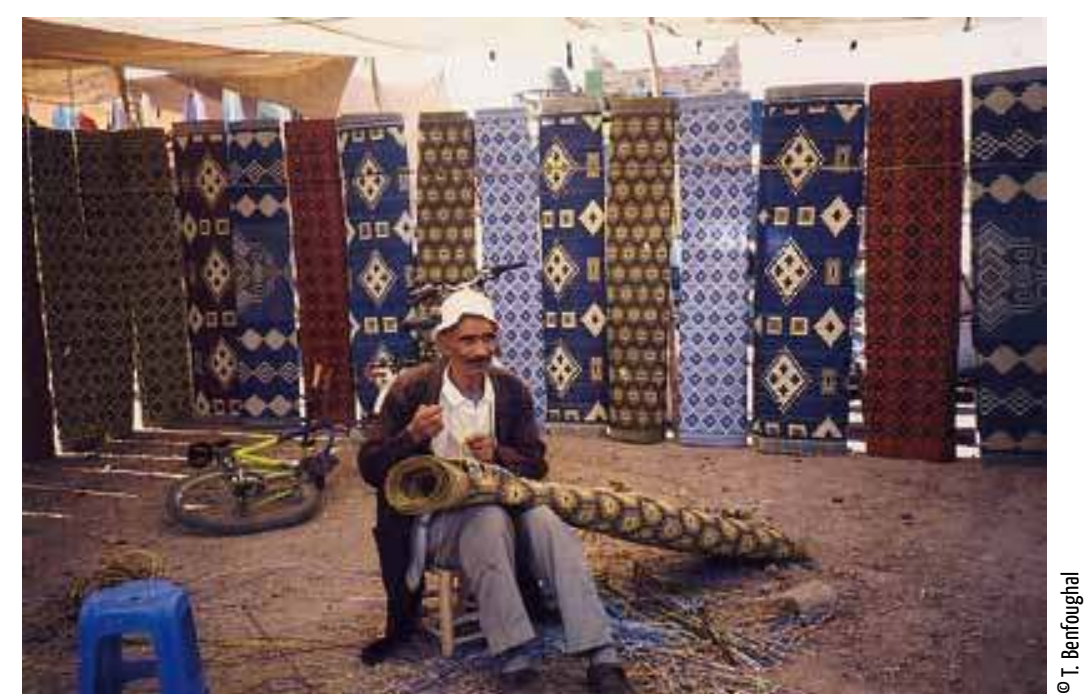

Pour répondre à la demande des femmes, les commerçants marocains vendent des lanières qu'ils récupèrent soit à la suite de la découpe des nattes (arrivant sur le marché en rouleau et vendues au mètre), soit en défaisant des portions entières de nattes. Maroc, vendeur des nattes au souk d'Ouarzazate, 2005.

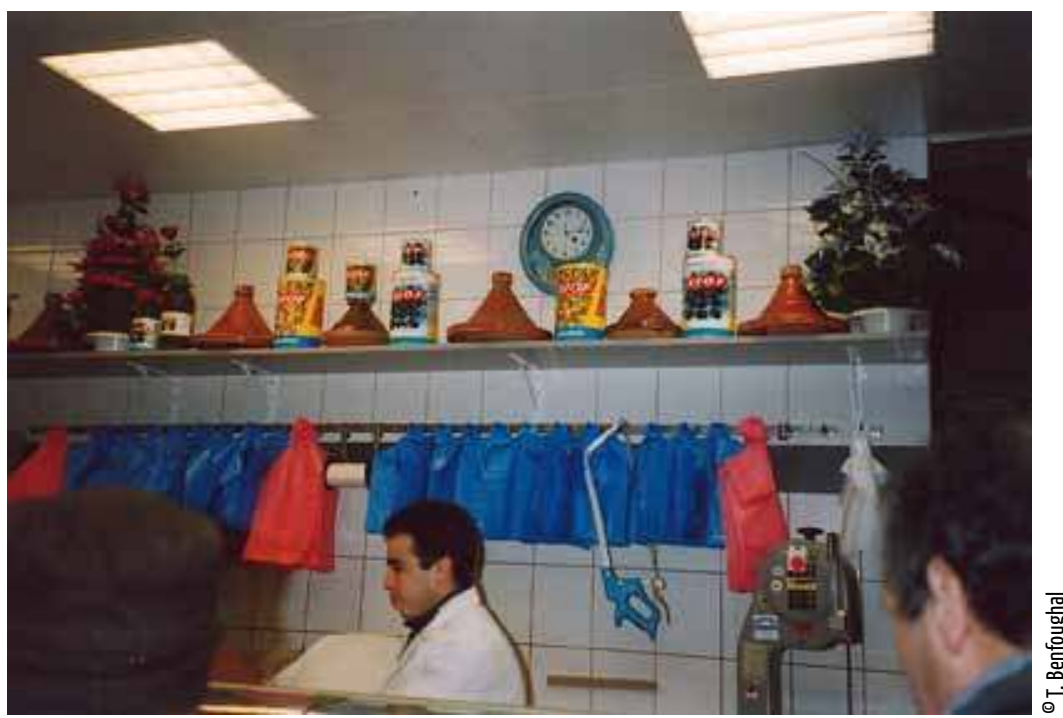

Les petits sacs du commerce de détail sont achetés par les femmes chez les épiciers pour quelques centimes. Maroc, Ouarzazate, 2002. jamais non plus ramassée dans la rue. Pour cette raison, il serait donc plus adapté, en parlant d'approvisionnement des matières plastiques dans les oasis, d'utiliser non pas le terme de « récupération », mais de « détournement ", dans le sens d'une soustraction de la matière à son usage prévu initialement.

\section{Moyens techniques de « métissage »}

Après le choix des plastiques à tresser, une autre démarche active dans le processus de métissage consiste en l'incorporation de la nouvelle matière dans le système technique endogène. En fonction du nombre et de la nature des éléments endogènes qui y participent (matières végétales, techniques, forme ou décor), plusieurs combinaisons avec la matière plastiques sont possibles.

La réalisation de ces combinaisons s'effectue toujours par le biais de réajustements et d'adaptations. Nous entrons là dans le domaine très riche des différents moyens d'« indigénisation créative » (Bromberger \& Chevalier 1999), qui va du recyclage culturel (changement de signification, de statut et de fonction de l'objet emprunté) au recyclage technique, compris comme un nouveau passage dans un cycle d'opérations et allant de différentes formes de «toilettage » de l'objet jusqu'à sa destruction, afin de récupérer les pièces détachées et la matière première (Benfoughal 2002).

L'utilisation des matières plastiques dans le tressage des vanneries représente le cas du recyclage de la 
matière première, lequel passe par deux phases : destruction de l'objet emprunté dont elle fait encore partie, puis son réemploi. Le tressage des vanneries ne pouvant se faire qu'avec des éléments longitudinaux, les femmes doivent couper des sacs et des sachets d'emballage en lanières. La recherche de solutions techniques de plus en plus efficaces constituant le moteur de toute démarche technique (Leroi-Gourhan 1992), les vannières cherchent la matière plastique déjà découpée et prête pour le tressage, en prélevant par exemple des lanières des nattes et des sacs tissés industriellement. Des solutions supplémentaires à la simplification des chaînes opératoires sont parfois apportées par des commerçants. Certains industriels mettent directement la matière première à la disposition des femmes, comme c'est le cas par exemple à la fabrique de nattes d'El Hamma, près de Gabès, où l'on vend aux femmes la matière plastique sous la forme de fils tubulaires, de couleurs et de métrages variables. Cette adaptation des « diffuseurs » maghrébins aux besoins locaux fait penser à un phénomène semblable relevé en Afrique du Sud où le succès des « vanneries » en fil téléphonique a amené l'entreprise de téléphonie de Johannesburg à produire des fils destinés spécialement aux artisans vanniers (Allen F. Roberts 2001). L’ultime étape de cette tendance à l'allégement de la chaîne opératoire du tressage est l'utilisation de bandes tissées industriellement que des femmes tunisiennes taillent et cousent abandonnant ainsi le métier de vannière et se transformant en « couturières ».

La deuxième phase du métissage technique consiste à incorporer des matières plastiques dans le tressage des vanneries. L'analyse que nous pouvons faire des modes de cette incorporation passe par la prise en considération des moyens locaux disponibles (outils, savoirs, procédés de tressage) et du degré de souplesse des chaines opératoires traditionnelles, capables ou non d'intégrer l'innovation. Effectivement, la matière plastique n'a pas provoqué la création de nouvelles chaînes opératoires, mais

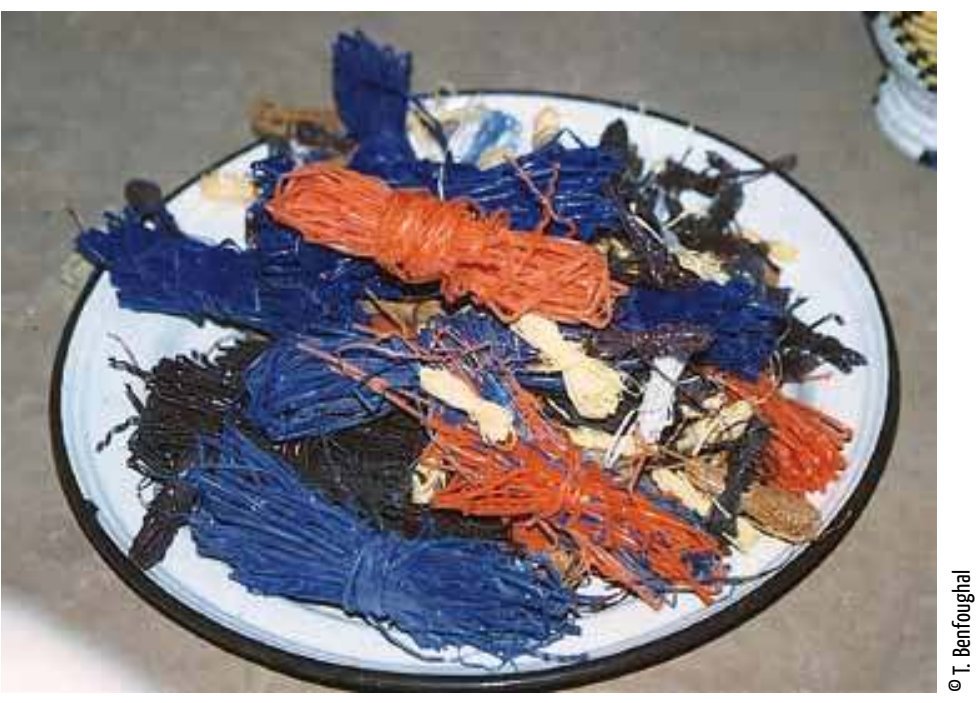
elle a été intégrée dans celles déjà utilisées dans le tressage des vanneries en fibres végéLanières destinées au tressage des vanneries. Maroc, vallée du Drâa, ksar Beni-Sbih. tales. Deux types de changements se sont produits néanmoins suite à son introduction. Le premier a consisté en un allégement de la chaine opératoire grâce à l'élimination de nombreuses phases de traitement des folioles de palmes habituellement utilisées, telles leur séchage pendant au moins une semaine, leur séparation en fines lanières, leur trempage dans l'eau pour les assouplir, puis plusieurs opérations de teinture (Benfoughal 2006). La matière plastique, elle, souple, teinte et souvent déjà en lanières, peut être tressée sans préparation particulière. Pour les femmes, cet allégement de la chaine opératoire constituait un des arguments en faveur de l'adoption de la matière plastique.

Un autre changement consistait en une modification partielle de la phase de tressage selon la technique du spiralé cousu. Si le tressage de la matière plastique selon les 


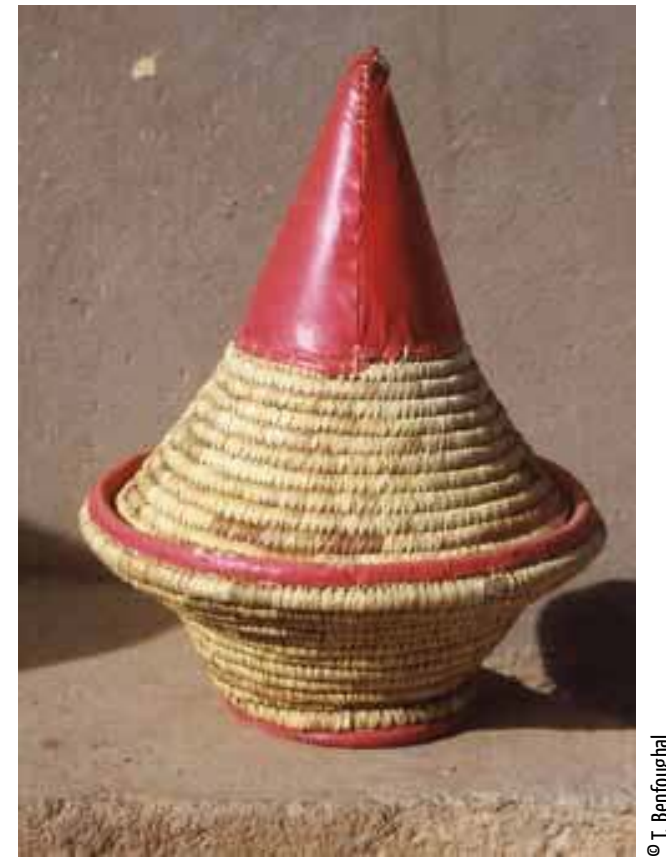

Dans cette variante de métissage, la vannerie garde la totalité de ses éléments endogènes (matière végétale, technique de tressage, forme du panier), la matière plastique n'apparaissant que sous la forme d’appliques ajoutées en surface. Maroc, vallée du Dadès, ksar Aït-Ouritane.

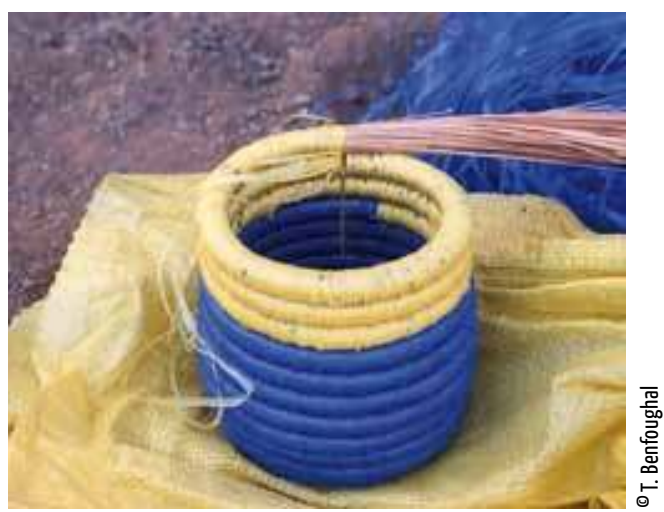

Cette variante de métissage, la plus fréquente à l'heure actuelle, concerne les vanneries tressées selon la technique du spiralées cousues où les brins, habituellement en folioles de palmier dattier, sont remplacés par des lanières de matière plastique. Celles-ci enveloppent le montant spiralé qui continue à être composé de tiges de la hampe du régime de dattes défibrée. Maroc, région de Foum-Zguid, Ksar Allogoum, 2002. techniques du tissé et du cordé, utilisées notamment pour fabriquer des nattes et des couffins, ne présentait pas de réelles difficultés, les gestes, les outils et les chaînes opératoires restant les mêmes, celle selon le spiralé cousu posait un problème technique : comment faire passer les brins en matière plastique, trop souple, dans les spires de la vannerie? Le procédé traditionnel, où le brin en lanière de foliole de palme affinée au bout est suffisamment rigide pour pénétrer dans l'orifice de la spire précédente, n'était plus envisageable dans le cas de la matière plastique. Le problème a été résolu par l'utilisation d'un outil nouveau, l'aiguille, avec quelques particularités suivant le pays. Par ailleurs, en Algérie et au Maroc, la technique du «noué », répandue dans les régions septentrionales du Maghreb et en Afrique subsaharienne, a fait son apparition car elle permettait de fixer les spires entre elles sans l'aide de l'aiguille.

Dans le cas de ces innovations techniques, la démarche novatrice consistait à établir des correspondances entre la matière plastique, la technique du spiralé et le procédé de couture. Cette mise en corrélation des trois composantes techniques (matière, besoin à satisfaire et moyen technique pour y parvenir) peut être considérée comme une invention, car elle propose, à partir d'éléments techniques disponibles, des solutions efficaces. Ces dernières sont trouvées non pas par la reproduction mécanique de quelques procédés, mais par des tâtonnements, des essais, des ajustements et des prises de décisions, aboutissant à la création de quelque chose de neuf, irréductible à la somme de ses composantes et qui constitue tout l'enjeu du métissage (Laplantine \& Nouss 1997 ; Bonniol 2001).

Nous aurions pu en rester là, sur ce constat de métissage technique novateur, si celui-ci n'avait pas été précédé d'une autre innovation, datant des années 1950, qui consistait en introduction dans le tressage, en qualité de brins, de fils de laine nécessitant le recours à l'aiguille et préparant ainsi le terrain sur le plan technique à l'introduction de la matière plastique. L'introduction des fils de laine n'a pas été due à la pénurie des matières végétales, ni à l'apparition soudaine de la matière laine, utilisée depuis longtemps pour les tissages oasiens, mais au changement de la fonction de certaines vanneries. Dans ce milieu du xxe siècle, afin d'enjoliver à la manière «moderne » les murs de leurs habitations, les femmes ont commencé à tresser des vanneries purement décoratives qui devaient être, par rapport aux vanneries « traditionnelles », plus visibles, plus attrayantes et plus « précieuses». L'introduction de la laine a répondu en fait à la nouvelle «tendance » comprise dans le sens d'A. Leroi-Gourhan 
(Leroi-Gourhan 1992 ; Martinelli 1993), où la fonction décorative des objets devait supplanter leur fonction utilitaire. Grâce à sa brillance et ses coloris vifs, la matière plastique satisfaisait encore plus efficacement la tendance enclenchée au milieu du $\mathrm{xx}^{e}$ siècle. Cette inscription de l'innovation dans un temps plus long, où l'on voit que le tressage des vanneries en matières plastiques a « récupéré » les moyens techniques mis en œuvre pour le tressage des vanneries en laine, renvoie à la question des limites du concept de métissage lequel suppose la mise en commun des « entités pures » alors qu'en réalité elles résultent elles-mêmes des hybridations antérieures (Amselle 2010).

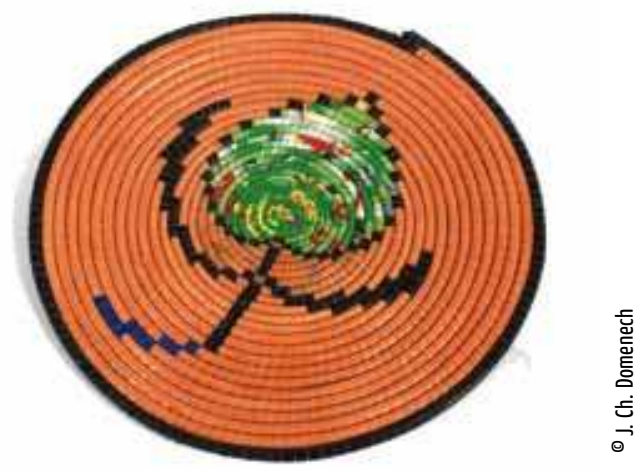

Les femmes algériennes ont tiré un profit esthétique d'un élément nouveau, le fil de nylon, visible sur la surface de la vannerie. Par un soin particulier apporté à l'alignement des points de couture et leur disposition en hélice, elles obtiennent un effet décoratif. Algérie, Tassili n-Ajjer, Djanet. n’ait intégré l'usage des matières plastiques dans les productions locales de tressage. Les liens, les cordes, les nattes, les couffins ou les corbeilles, fabriqués en plastique, font désormais partie du quotidien des Asiatiques, des Amérindiens ou des Africains. Multiples sont les raisons de cette adoption pour laquelle les qualités du matériau, à savoir sa plasticité et sa solidité jouent un grand rôle. Mais ces raisons pratiques ne sont pas les seules. D’autres s'y ajoutent, en fonction des contextes géographiques, économiques et socio-culturels propres à chaque culture et à chaque communauté.

En Europe, l'argument écologique est souvent le premier à justifier la récupération et le recyclage des matières plastiques. Dans les pays en voie de développement, et plus particulièrement dans les pays africains, c'est la raison économique qui prime, même si les considérations écologiques peuvent y être adjointes. La motivation économique du recyclage a été clairement exprimée lors de la Table ronde « Les métiers du recyclage et leurs potentiels d'insertion socio-économique » organisée au Maroc en 1998 : « Les activités de récupération ne sont pas conçues par un intérêt environnemental mais plutôt économique »

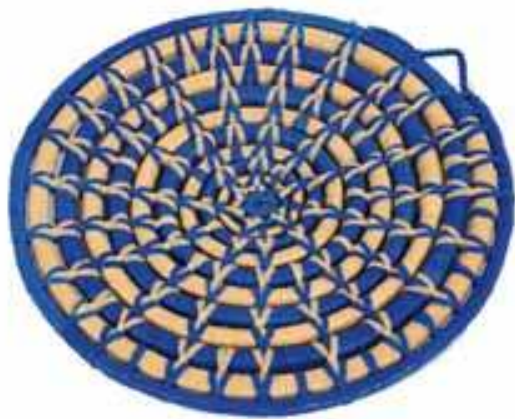

(Diallo A. Amadou 1998). Cette motivation économique était souvent la seule à être considérée dans un grand nombre d'analyses des artisanats des pays en voie de développement, comme celui dite de tanake et pour lequel la pénurie et la misère ont été évoquées comme uniques moteurs de son développement (Varine 1978). Pour contredire cette affirmation, les auteurs africains participant au catalogue de l'exposition «Ingénieuse Afrique » organisée en 1994 à Québec, mettaient en lumière une autre façon de "récupérer » et de « recycler » dans les pays du tiersmonde, celle des artistes africains contemporains (Souza Ayari 1994).
La technique du spiralé noué permet d'obtenir un effet esthétique : passé puis noué à distances régulières, le brin forme un décor radiaire ou en hélice, d'autant plus marqué si le brin est noué à point fondu. 
Entre ces différents arguments, écologiques, économiques ou artistiques, la fabrication des vanneries sahariennes en matière plastique ne semble pas trouver son explication. Elle ne relève pas du souci environnemental, car l'écologie n'est pas encore une priorité pour de larges couches de la population oasienne. Elle ne répond pas, ou très peu, aux besoins économiques, car elle est très peu destinée à la vente, avec, de surcroît, une matière achetée, alors que celle tirée du palmier dattier est gratuite et disponible. Elle n'est pas destinée non plus aux galeries d'art.

Pour essayer d'entrevoir les raisons de l'utilisation de la matière plastique dans les vanneries sahariennes, il faut que nous considérions tout d'abord la gamme des objets qui en sont fabriqués et l'usage que les Sahariens en font. Il se trouve qu'en plus des nattes et des couffins, que l'on achète le plus souvent dans le commerce et pour lesquels l'utilisation du plastique est motivée en grande partie par sa solidité, les femmes tressent des vanneries qui sont utilisées notamment lors des fêtes et des repas collectifs ou qui sont exposées dans la pièce principale de la maison bien en vue, sur une étagère ou accrochées au mur. Ce sont des plats dans lesquels on propose aux convives dattes, biscuits, cacahuètes ou bonbons; des couvre-plats qui protègent la nourriture chaude contre le sable et les « mauvais esprits»; des paniers à couvercle qui contiennent le pain ou les dattes.

Vanneries en matière plastique provenant d’Afrique, du Moyen-Orient et des régions septentrionales du Maghreb

Niger (deux vans et un panier, rangée verticale, à gauche), Guinée (un plat, rangée du bas, au centre, et un panier, au-dessus), Turquie (plat, rangée du bas, à droite), Algérie, Grande Kabylie (deux cruches assemblées, rangée du haut, à droite, et une corbeille, au-dessous), Algérie, région de Msila (deux corbeilles, rangée du haut, au centre, et rangée du milieu, au centre).

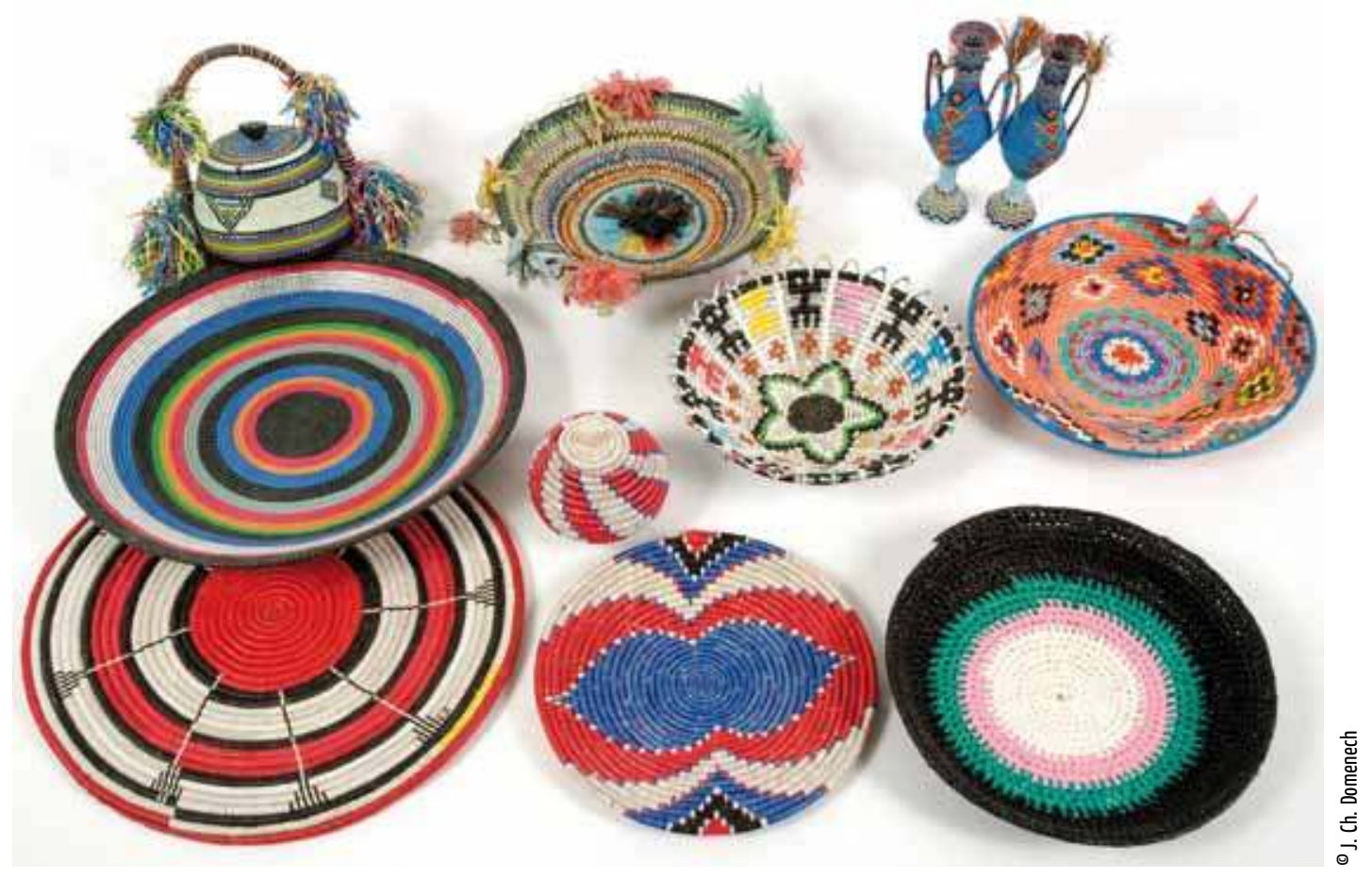


Vu le contexte de leur utilisation, on peut supposer d'amblée que le rôle des vanneries en matières plastiques n'est pas qu'utilitaire. Leur aspect décoratif est très remarqué, grâce notamment aux coloris vifs ainsi qu'à l'effet de brillance apprécié localement. Ce dernier s'inscrit dans le système des anciennes pratiques liées au mauvais œil, car considéré comme efficace pour neutraliser l'effet néfaste de celuici. Sur ce plan, "l'esthétique du brillant» n'est pas vraiment nouvelle au Maghreb et au Sahara, comme on a l'habitude de dire (Puig 2003 : 256 ; Gosselain \& al. 2008 : 23). Elle est connue par ailleurs un peu partout dans le monde, comme par exemple chez les Sulka en Papouasie-Nouvelle-Guinée ou chez les Amérindiens de la côte Nord-Ouest qui apprécient les qualités des matières plastiques, exprimées localement en termes d'éclat, de brillance et de clarté, puisque correspondant à la conception locale du beau et sa liaison étroite avec les notions esthétiques de « frais », de « récent » et de «neuf» (Jeudi-Ballini 1999 ; Mauzé 1999). L'aspect «neuf » des vanneries en plastique, que les femmes sahariennes cherchent toujours à leur donner, renforce leur fonction décorative. Mais il correspond, lui aussi, aux normes esthétiques traditionnelles, se rapportant plus particulièrement aux vêtements, tapis et nombreux objets domestiques, surtout ceux destinés aux cadeaux de mariage. Pour que les vanneries aient toujours un aspect neuf et brillant, les femmes renouvellent leur stock aussi souvent que possible. Dans ce contexte, le caractère non périssable de la matière plastique n'entre pas pour elles en ligne de compte et ne constitue pas par conséquent un facteur déterminant de son utilisation.

Les vanneries en plastique rejoignent par leur fonction décorative de nombreux objets d'artisanat traditionnel, mais elles aussi ont une valeur ajoutée. Celle-ci apparaît à travers la manière dont elles sont exposées dans l'espace domestique : à côté du poste de télévision, d'un ventilateur, d'un sèche-cheveux ou d'un magnétoscope, tous ces objets qui « viennent d'ailleurs » (Benfoughal 2002) et qui sont là non seulement pour servir et enjoliver la maison mais aussi et surtout pour signifier richesse et pauvreté. Dans les années 1970, au début de l'introduction de la matière plastique et du papier aluminium, sous la forme d'emballages de produits importés de l'étranger ou des grandes villes maghrébines, ceux-ci arrivaient dans les oasis investis d'une valeur associée au statut privilégié de personnes qui pouvaient voyager et acheter les produits industrialisés (immigrés, commerçants, fonctionnaires). Les vanneries en matières plastiques participaient ainsi, en tant qu'objets de prestige, à la « mise en objets de la divergence sociale » (Warnier 1999a : 119) et à la reconnaissance
Fillettes avec paniers pour hammam tressés en papie aluminium. Algérie, vallée de la Saoura, Ksar Zaouia Tahtania, 1990. 
identitaire de ceux qui « avaient de l'argent ». Puisque signifiant la réussite personnelle et un statut privilégié de leur propriétaire, en dehors de son appartenance statutaire traditionnelle, elles devenaient valorisantes et valorisées.

$\mathrm{Au}$ fur et à mesure que la matière plastique devenait accessible, elle perdait de son prestige social, mais gagnait celui de la modernité. Selon les dires des femmes, les vanneries en matière plastique sont 'asri (arabe), « modernes», par opposition aux vanneries en matières végétales, taqlidiyat (arabe), «traditionnelles». Pour mesurer la force de ce prestige culturel, il faut se mettre à la place du Saharien et essayer de comprendre sa perception de la modernité. Les premiers éléments de réponse sont donnés par les femmes. La matière plastique évoque pour elles les produits fabriqués industriellement, les villes, les grands magasins et l'abondance. Son aspect lisse, brillant et coloré renvoie aux images des belles robes et des riches maisons des séries télévisées et des magazines. Sa valeur marchande suggère l'argent, le travail salarial et l'économie monétaire moderne. Le fait de devoir et de pouvoir l'acheter est considéré par les femmes comme un signe d'ascension sociale. Son caractère de matière "étrangère » a aussi son importance, car aux yeux des consommatrices, elle prouve leur ouverture sur le monde extérieur et leur acceptation du progrès. Bien que les centres de production du plastique se trouvent maintenant à la porte des oasis, cette matière continue à être considérée par les oasiens comme culturellement exogène puisque relevant des technologies et des savoirs venant « d'ailleurs » et ne relevant pas de l'économie traditionnelle. Les appellations en un français « domestiqué » de nilou (déformation du mot nylon), de lahda (marque de lait en poudre, vendu dans les sachets de polyéthylène adhéré sur le papier aluminium), de mika (mica, substance minérale à éclat métallique) ou de fil-kado («fil cadeau », rubans pour paquets cadeaux) confirment aux yeux de tous, l'origine étrangère de la matière plastique. Appliquées par extension aux vanneries, elles témoignent des nouvelles identités que les femmes veulent leur donner.

Si l'industriel, l'acheté et le « venu d'ailleurs » sont perçus par les Sahariens comme différentes facettes de la modernité, c'est parce que celle-ci, loin de sa signification historique initiale, leur apparaît dans sa matérialité technique quotidienne (Baudrillard 1985), souvent d'origine occidentale. L'électricité, les routes goudronnées, les villes, les puits de pétrole, l'argent et les produits manufacturés sont pour les Sahariens ses éléments les plus visibles. Cette lecture locale de la modernité, comme étrangère à leur propre culture mais impliquant le changement de leur mode de vie et de leur mentalité, conduit les Sahariens au renoncement à certaines traditions ancestrales et les met devant un choix culturel entre la «tradition » et la «modernité », le passé et le présent. Le caractère exclusif de ce choix, de caractère identitaire, s'explique en grande partie par l'histoire récente du Sahara maghrébin. D'abord, par l'occupation française, dès le début du xxe siècle, avec ses bouleversements multiples dont la sédentarisation, la scolarisation, l'industrialisation, l'urbanisation, etc. Pour réaliser cette « modernisation » du Sahara, le pouvoir colonial devait casser les anciens systèmes socio-économiques basés sur la libre circulation des nomades, sur la complémentarité des nomades et des sédentaires, sur les hiérarchies tribales complexes, sur le respect des normes éthiques traditionnelles, tous ces fondements qui étaient incompatibles avec le mode de vie que la puissance dominante a voulu imposer. Pour les faire accepter aux Sahariens, il a fallu prétendre qu'être sédentaire était mieux qu'être nomade, qu'être salarié était plus avantageux financièrement qu'être agriculteur, que parler le 
français était mieux que parler l'arabe ou le berbère, que vivre dans une maison en ciment était mieux que dans une maison en terre, etc. La fracture s'est opérée progressivement entre les deux modes de vie et les deux manières de penser : le traditionnel devenant négatif et rétrograde, alors que tout ce qui était « nouveau » et imposé par le colonisateur était présenté comme positif, significatif du progrès et « civilisé ». La même vision antagoniste a été perpétuée et même accentuée par les états du Maghreb dès leurs indépendances, avec cette seule différence que le monde « civilisé » n'était plus limité à la France et aux autres pays occidentaux mais couvrait aussi l'espace économique et culturel étatique des pays maghrébins. Cette vision s'est emparée aujourd'hui de nombreuses régions sahariennes et plus particulièrement des milieux sédentaires ${ }^{9}$. Le récent développement du tourisme national et international, surtout au Maroc et en Tunisie, les flux migratoires et l'apparition de structures commerciales modernes ont mis les Sahariens en présence de ces modes de vie et mentalités « occidentaux », enviés et perçus comme des modèles à suivre. Aujourd'hui, la plupart des familles sahariennes sédentaires veulent bénéficier des avantages du monde moderne et, en ce qui concerne le côté proprement matériel, vivre «à la citadine ». La télévision, le mobilier, les bibelots et les vanneries « modernes » commencent à faire partie de leur quotidien, comme des signes de cette nouvelle culture de référence, luxueuse, citadine, industrielle et occidentale.

\section{$\&$}

L'analyse que nous venons de faire, démontre que la manière dont la matière plastique a été introduite dans le tressage des vanneries constitue une réponse au nouveau contexte socio-économique et culturel au Sahara et, plus largement, en Afrique. Avec les démarches de patrimonialisation ou de folklorisation, elle s'inscrit dans l'ensemble des stratégies que les cultures locales proposent pour s'associer à la modernité sans pour autant rompre le fil qui les relie à la «tradition». Néanmoins, par rapport aux deux premières, qui ont l'inconvénient de figer les pratiques en les mettant en scène ou en vitrine, elle contribue à leur pérennisation en les ouvrant aux innovations. La vannerie «métissée » permet ainsi de faire perdurer certains éléments traditionnels (techniques, formes fonctionnelles et décor) et de satisfaire l'aspiration à la modernité. Elle permet aux femmes de s'associer à la modernité d'une façon active, c'est-à-dire non pas de la subir, mais de construire à partir des emprunts et par des associations inventives quelque chose qui leur est propre.

Nous avons vu aussi que cette emprise active sur le plastique se produit en fonction des traditions techniques locales et des possibilités d'approvisionnement que chacun des trois états offre, donnant naissance aux nombreuses variantes régionales des vanneries. Je n'ai pu me rendre compte de cette diversification, qui a atténué la tendance à l'uniformisation et au nivellement stylistique, constaté dans ma première étude, qu'après une analyse comparative dans les trois pays maghrébins. L'exemple de la vannerie "métissée » saharienne confirme ainsi la thèse selon laquelle la mondialisation, tout en provoquant l'effritement des traditions, n'est pas forcément 
synonyme de convergence des cultures du monde vers un modèle unique, mais, au contraire, qu'elle est une formidable machine à produire de la différence culturelle (Warnier 1999b). Ceci parce que les cultures locales résistent à la déconstruction et à l'uniformisation. Parce qu'elles sont toujours capables d'imagination, de création et d'inventivité. Et parce que les industries modernes leur proposent des produits de plus en plus nombreux et diversifiés. Les vanneries en nilou, lahda, mika et fils $k a d o$, versions contemporaines des vanneries sahariennes, sont l'expression de cette modernité multiple et une réponse culturellement personnalisée à la mondialisation et à la globalisation.

\footnotetext{
Salon d'une maison dans le ksar AïtYacine, région de Tata, Maroc. 2002

Parmi les objets exposés, on remarque un téléviseur, une chaîne Hi-Fi, un poste radio, trois ventilateurs, un service à thé en inox, plusieurs vases contenant des fleurs en matière plastique. Sur chacune des deux tables basses, de part et d'autre l'étagère centrale, est posé un grand panier en matière plastique tressé par les femmes de la maison. Ce type de panier, quand il est tressé en matières végétales, sert à contenir une provision de pains. Ici, parmi d'autres objets « venus d'ailleurs », ils remplissent une fonction décorative et ostentatoire.
}

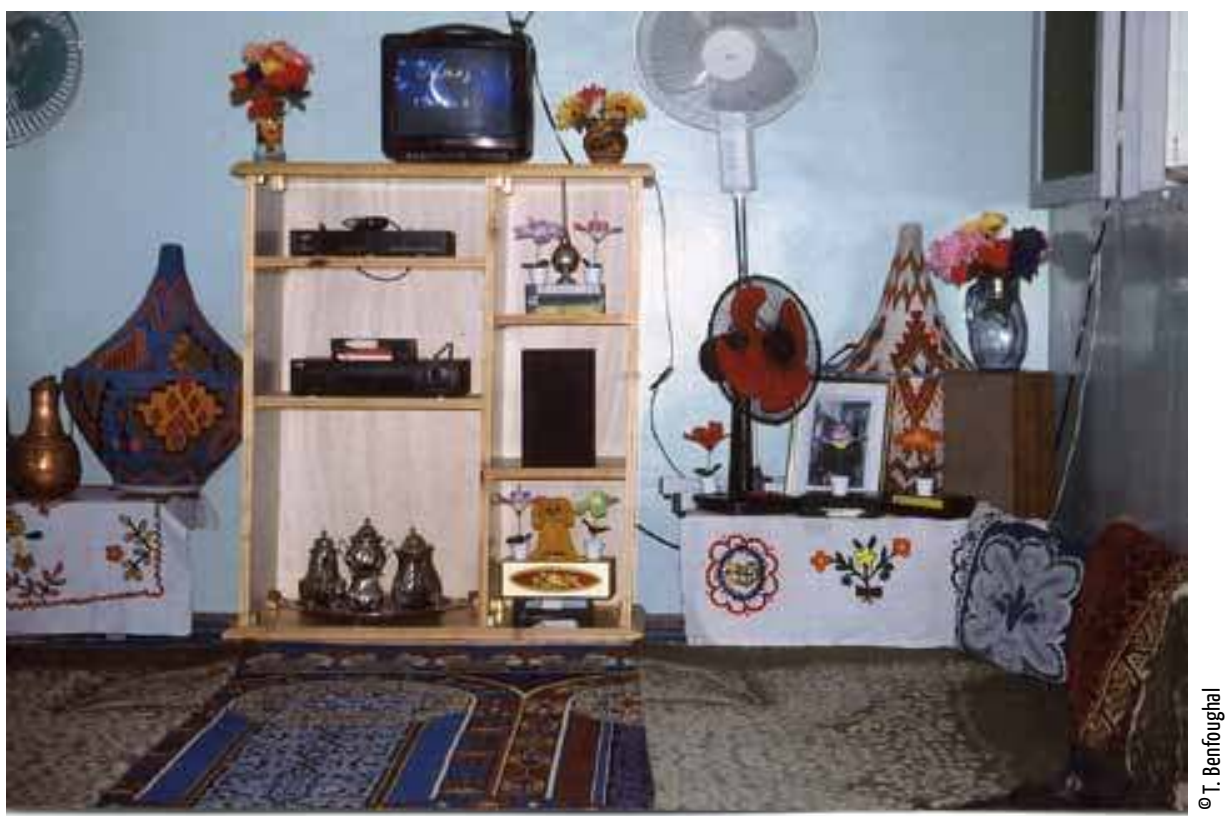




\section{NOTES}

Photo d'ouverture: Les sacs de polyéthylène issus du commerce de détail sont coupés en lanières, lesquelles sont utilisées dans le tressage des vanneries selon la technique du spiralé cousu.

1. Ce texte fait suite à un article consacré à l'esthétique des vanneries sahariennes et publié dans le n51 de Techniques et Culture (Benfoughal 2008).

2. Dans les oasis algériennes par exemple, le prix moyen d'un plat en matière plastique s'élevait en 1990 à 150 dinars, alors que celui en palmier dattier n'était que de 75 dinars.

3. Voir notamment Leroi-Gourhan (1992), Hainard \& Kaehr (1984), Segalen \& Bromberger (1996), Dionne, Claude \& al. (dir.), 1996, Bromberger \& Chevallier (1999), Bonnot (2002), Benfoughal \&t Boulay (2006), Kopytoff (2006), Gosselain \& al. (2008), Wateau (2011).

4. Pour la terminologie concernant les techniques de tressage voir Benfoughal, 2006

5. Parmi quelques-unes on peut mentionner la «Société maroco-syrienne de tapisserie en plastique » (SAMOSYT) de Berrechid, la «Société tunisienne des nattes en plastique » de Naassen, celle de Ben Arous Ben Arous, ainsi que plusieurs usines des nattes en Algérie : à Alger, à Bejaia, à Annaba, à Sétif, à Hippone, etc. Pour l'Afrique sub-saharienne, la plus fameuse est la « Société Sénégalaise de Nattes en Plastiques » (SO.SE.NA.P) de Dakar.
6. Je remercie le professeur Bernadette Charleux et son équipe du Laboratoire de chimie des polymères de l'université Pierre et Marie Curie pour l'identification des échantillons de matières plastiques que je leur ai soumis.

7. À titre d'exemple, on peur citer l'entreprise « Lalla Mika » de Marrakech ou encore " Qui dit mieux » de Porto-Novo au Bénin.

8. Pour les matières plastiques, les déformations mécaniques (étirage, pliage, etc.) et chromatiques (perte de couleur sous l'effet de la lumière) sont de nature irréversible.

9. Pour les Touaregs nomades, leur perception de « modernité » et de « civilisation» est moins positive. Au Niger, par exemple, l'expression tantut da sibilise a tesa (" cette femme-là est civilisée ») a une connotation péjorative. Les filles et les garçons « civilisés », que l'on nomme aussi « ceux de la ville » (ag ajrem et welet ajrem) sont habituellement mal vus (communication personnelle de Saskia Walentowitz et Abdoulmohamine Khamed Attayoub). 


\section{RÉEÉRENCES}

Allen F. Roberts 2001 Break the silence, African arts, volume XXXIX : 37-49.

Amselle, J.-L. 2010 [1990] Logiques métisses. Paris : Petite Bibliothèque Payot.

Barthes, R. 1967 Système de la mode. Paris : Éditions du Seuil.

Baudrillard J. 1985 Modernité, Encyclopaedia Universalis, Tome 12 : 424-426.

Benfoughal, T. 1996 Du Palmier dattier à la matière plastique. Tradition et mode dans la fabrication des vanneries sahariennes, Les cahiers de l'IREMAM 7-8: 57-78.

- 2002, Ces Objets qui viennent d'ailleurs, Les cahiers de l'IREMAM : 113-134.

— 2006 De la matière première à l'objet tressé. La vannerie dans les oasis du Sahara maghrébin, Journal des Africanistes 76 : 141-164.

— 2008 Qu'est ce qu'une belle vannerie au Sahara? Normes collectives et choix individuels, Techniques \& culture $51: 217-245$.

Benfoughal, T. \& Boulay, S. (dir.) 2006 Sahara : identités et mutations sociales en objets, Journal des Africanistes 76.

Bonniol, J.-L. (dir.) 2001 Paradoxes du métissage. Paris : Éditions du CTHS.

Bonnot, T. 2002 La vie des objets. Paris: Éditions de la Maison des sciences de l'homme.

Bromberger, C. \&Chevalier, D. (dir.) 1999 Carrières d’objets. Paris:Édition de la Maison dessciencesdel'homme.

Diallo A. Amadou 1998 L'Artisanat utilitaire et artistique : un état des lieux à travers une expérience de l'Afrique subsaharienne. In M. Ftouhi (dir.), Les métiers du recyclage et leurs potentiels d’insertion socioéconomique, Table ronde. Site web [consulté le 11/06/12] : http://www.globenet.org/preceup/pages/fr/ chapitre/capitali/cas/recy_b.htm

Dionne, C. \& al. (dir.), 1996 Recyclages. Économies de l'appropriation culturelle. Montréal : Éditions Balzac.

Gosselain, O., Zeebroek, R. \& Decroly, J.-M. 2008 Des choses, des gestes, des mots. Repenser les dynamiques culturelles, Techniques \& culture 51.

Gruzinski, S. 1999 La Pensée métissée. Paris : Fayard.

Hainard,J. \&Kaehr, R. (dir.) 1984 Objetsprétextes, objetsmanipulés. Neuchâtel:Muséed'Ethnographie de Neuchâtel. Jeudi-Ballini, M. 1999 Dédommager le désir. Le prix de l'émotion en Nouvelle-Bretagne (PapouasieNouvelle-Guinée), Terrain $32: 5-20$.

Kopytoff, I. 2006 [1986] La Biographie culturelle des choses, Journal des Africanistes 76 : 217-250.

Laplantine, F. \& Nouss, A. 1997 Le Métissage. Paris : Flammarion.

Leroi-Gourhan, A. 1992 [1945] Milieu et technique. Paris : Albin Michel.

Martinelli, B. (dir.) 1993 Atouts et outils de l'ethnologie des techniques. Sens et tendance en ethnologie comparée, Techniques \& culture 21.

Mauzé, M. 1999 L'Éclat de l'haliotide. De la conception du beau dans les sociétés de la côte Nord-Ouest, Terrain $32: 83-98$.

Puig, N. 2003 Bédouins sédentarisés et société citadine à Tozeur (Sud-ouest tunisien). Paris : IRMC-Karthala.

Rouillard P. 2007 Mobilités, immobilismes. L'emprunt et son refus. Paris : Éditions De Boccard.

Segalen, M. \& Bromberger, C. (dir.) 1996 Culture matérielle et modernité, L’Ethnologie française 26.

Souza Ayari (de), R. 1994 Récupération et créativité. In Ingénieuse Afrique. Artisans de la récupération et du recyclage, catalogue d'exposition du Musée de la civilisation, Québec.

Turgeon L. 2003 Patrimoines métissés. Contextes coloniaux et postcoloniaux. Paris : Éditions de la Maison des sciences de l'Homme; Québec: les Presses de l'université Laval.

Varine (de), H. 1978 Le Tanaké, découverte d'une culture. In Fer blanc et fil de fer, Catalogue d'exposition du Centre Georges Pompidou : 11-14.

Warnier, Jean-Pierre 1999a Construire la culture matérielle. Paris : PUF.

- 1999b La Mondialisation de la culture. Paris : Éditions La Découverte, Syros.

Wateau, F. (dir.), 2011 Profils d’objets. Approches d'anthropologues et d'archéologues. Paris : De Boccard. 


\section{RÉSUMÉ}

La vannerie saharienne se métisse. Les vanneries en matière plastique, fabriquées aujourd'hui dans les oasis du Sahara maghrébin, sont à la croisée des traditions locales et des emprunts « modernes ». Elles représentent l'un des éléments du processus d'interactions culturelles plus globales, entre le monde saharien et le monde occidental, l'un de ces « entre-deux » où s'effectuent les métissages des matières, des savoirs techniques, des normes esthétiques et des usages des objets fabriqués. Du fait du choix par la population locale des matières plastiques disponibles, du fait des processus de «déconstruction » des objets d'origine occidentale ainsi que de l'introduction dans le tressage des matières ainsi extraites, la production vannière actuelle est considérée par l'auteur comme l'une des réponses actives des Sahariens à la pression du monde moderne.

\section{ABSTRACT}

Saharan basketwork gets crossbred. Plastic baskets, today made in the oasis of Maghreb Sahara, are at the crossroads of local traditions and « modern » borrowings. They represent one element of the global process of cultural interactions between Saharan world and Western world, one of these «two » into which any interbreeding of materials, technical knowledge, aesthetic standards and practices artifacts take place. Because of the choice by the local population of available plastics, because of the " deconstruction » of objects of Western origin and the introduction of these extracted materials in braiding, basketwork current production is considered by the author as one of active responses of Saharan people to pressure of modern world.

\section{MOTS-CLÉS}

Sahara, oasis, vannerie, matière plastique, métissage.

\section{KEYWORDS}

Sahara, oasis, basketry, plastic, crossbreeding. 